\title{
DINER (Data Into Nutrients for Epidemiological Research) - a new data-entry program for nutritional analysis in the EPIC-Norfolk cohort and the 7-day diary method
}

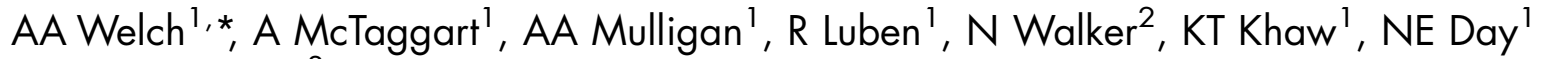 \\ and SA Bingham ${ }^{3}$ \\ 'University of Cambridge, Institute of Public Health, Strangeways Research Laboratory, Wort's Causeway, \\ Cambridge CB1 8RN, UK: ${ }^{2}$ JDRF/WT Diabetes and Inflammation Laboratory, University of Cambridge, Cambridge \\ CS2 2XY, UK: ${ }^{3}$ Dunn MRC Human Nutrition Unit, Cambridge, CB2 2XY, UK
}

Submitted 30 March 2001: Accepted 22 May 2001

\begin{abstract}
Background and objective: A new data-entry system (DINER - Data Into Nutrients for Epidemiological Research) for food record methods has been devised for the European Prospective Investigation into Cancer (EPIC) cohort study of 25000 men and women in Norfolk. DINER has been developed to address the problems of efficiency and consistency of data entry, comparability of data, maximising information and future flexibility in large long-term population studies of diet and disease that use record methods to assess dietary intakes. DINER captures more detail than traditional systems and enables provision of new variables for specific food types or groups. The system has been designed to be fully flexible and easy to update. Analysis of consistency of data entry was tested in a group of 3525 participants entered by 25 coders.

Results: A food list of 9000 food items and values for 24000 portion sizes have been incorporated into the database, using information from the 5979 diaries coded since 1995. Analysis of consistency of entry indicated that this has largely been achieved. The effect of coders in a multivariate regression model was significant only if the three coders involved in early use of the program were included $(P<0.013)$. Conclusions: The development of DINER has facilitated the use of more accurate record methods in large-scale epidemiological studies of diet and disease. Furthermore, the retention of original information as an extensive food list allows greater flexibility in later analyses of data of multiple dietary hypotheses.
\end{abstract}

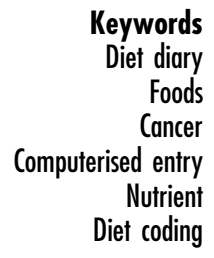

Accurate estimation of nutritional intake is fundamental to nutritional epidemiology. There are various methods for the assessment of dietary intake in free-living individuals. The two main approaches are food-frequency questionnaires (FFQs), which have pre-assigned lists of foods and frequencies of consumption, and diet records, which allow open-ended listing of food intake. All methods are associated with errors of differing magnitude. The introduction of biomarkers has allowed these errors to be assessed. Recent studies have shown that food record methods have closer associations with biomarkers and reduced measurement error - both random and, more importantly, systematic errors - when compared with other dietary instruments ${ }^{1-4}$. Because food records are open-ended, they are more flexible and able to capture a much wider variety of foods and nutrients, increasing potential accuracy. However, practical and methodological difficulties have limited the use of record methods in large-scale studies, most notably the difficulty in coding and data entry of food intake for nutrient analyses. The accuracy of nutrient intake from record methods is dependent on the precision and completeness of the data collection, the accuracy and representativeness of the food composition database used, and the consistency and precision when translating records during entry.

The European Prospective Investigation into Cancer (EPIC) is a prospective study of diet and cancer in 400000 men and women aged $30-75$ in nine European countries $^{5}$. The Norfolk component of this collaboration, EPIC-Norfolk, comprising 25000 men and women aged 40-75 years first surveyed in 1993-1997, has been extended to enable study of other chronic diseases including cardiovascular disease, diabetes and osteoporosis $^{6}$.

This paper describes the rationale behind the 
development of the DINER (Data Into Nutrients for Epidemiological Research) program used for entry of 7 day diaries (7D diaries) in the EPIC-Norfolk cohort and gives details of the diary method. DINER can also be used for entry of 24-hour recalls $(24 \mathrm{H})$ and weighed records. Whilst developed for use within the United Kingdom for a large-scale study, the principles have wider application in the field of nutritional epidemiology and surveillance.

\section{Methods}

\section{Rationale}

The identification and classification of reported foods and the association with the amount consumed are central to the data-entry process. The DINER program aids the translation from reported free text to structured data, which are then converted into food and nutrient data for analysis.

Although entry systems are important tools underpinning nutritional epidemiology, the systems available in 1993 were similar and had not been developed to support the needs of a large-scale prospective cohort study with an extended period of data collection and entry. A system was needed that would be sufficiently robust to deal with the anticipated changes that would occur to foods and satisfy the multiple hypotheses to be studied.

Processing of food records for large-scale studies introduces problems of scale, access to information and comparability of data not apparent for smaller studies. As food record data require interpretation during entry, they are more sensitive to the influence of and method of use of the entry system than other more structured methods such as FFQs. Treatment of many thousands of diaries over several years by numerous staff could have created sources of bias unless attention was paid to consistency. Study-specific bias had to be avoided as data were to be entered for a variety of different disease endpoints. Rapid and efficient entry was also essential.

Record methods provide detailed information on consumption of food type and amount, but this advantage is lost unless captured electronically. DINER has been developed to record in a form as close as possible to the original by incorporating a detailed classification system. Systems in 1993 were unable to capture sufficient detail as they were based on the limited number of food types available within food composition databases. The range of published portion sizes was also limited. Detailed entry enables creation of new variables for specific food types or groups in response to the burgeoning number of hypotheses relating diet to disease. Detail makes the system flexible and easy to update, an important consideration as entry costs are high and would become prohibitive if the original data required re-entering for updating purposes. In addition, EPIC-Norfolk incorporates follow-up investigations of the cohort participants, whereas the data management characteristics of the systems available in 1993 were inadequate for a largescale study with repeated dietary measurements.

\section{Dietary methods}

All EPIC-Norfolk participants completed an initial selfreported $24 \mathrm{H}$, a $7 \mathrm{D}$ diary and an FFQ. For follow-up, repeats of the $24 \mathrm{H}$ and $7 \mathrm{D}$ diary were obtained at 18 months and 4 years. The FFQ and self-reported $24 \mathrm{H}$ were completed by participants before attending a clinic where they were instructed by nurses on how to complete the $7 \mathrm{D}$ diary. As part of this instruction, a $24 \mathrm{H}$ formed the first day of the first 7D diary and recorded the period from waking to sleeping on the previous day. This demonstrated the process of recording intake and indicated the level of detail required. Recording in detail is important as this improves precision of the method and reduces problems of interpretation and ambiguity during data entry. A carbon copy of this interview was retained in the event that the diary was not completed and returned to the EPIC centre.

All interviewers were trained to a standardised technique, using a formal documented protocol. The intention was to reduce the potential for the impact of the interview on the reporting process. Specific advice was given so that the expression of criticism, surprise, disapproval or praise was avoided. Interviewers were requested not to ask 'leading questions' or give dietary advice. Neutral probing questions were used to elicit detail of foods consumed. At the end of the interview, interviewers probed for foods that might have been omitted by using a checklist of foods and snacks. Participants completed the remaining 6 days and returned the diary by post.

The diary was based on that developed for the MRC Study of Health and Development ${ }^{7}$. It consisted of instructions to guide the detail and type of information to be reported and suggested methods for estimating the amount of food consumed, such as household measures, recognisable standard units or the series of 17 colour photographs representing small, medium or large portions. Consumption was reported in a series of seven meal slots with space for additional snacks and other information.

At the end of the week's record additional questions were completed on important contributors to nutrient intake such as milk, bread and fat consumption to clarify the information already supplied. Consumption of vitamin and mineral supplements was recorded and entered separately for later analysis.

\section{Overview of the DINER system}

Figure 1 shows an example of information obtained from the 7D diary. The purpose of the DINER program was to convert reported data into structured data files (DINER files) and final nutritional analysis. A schematic overview 


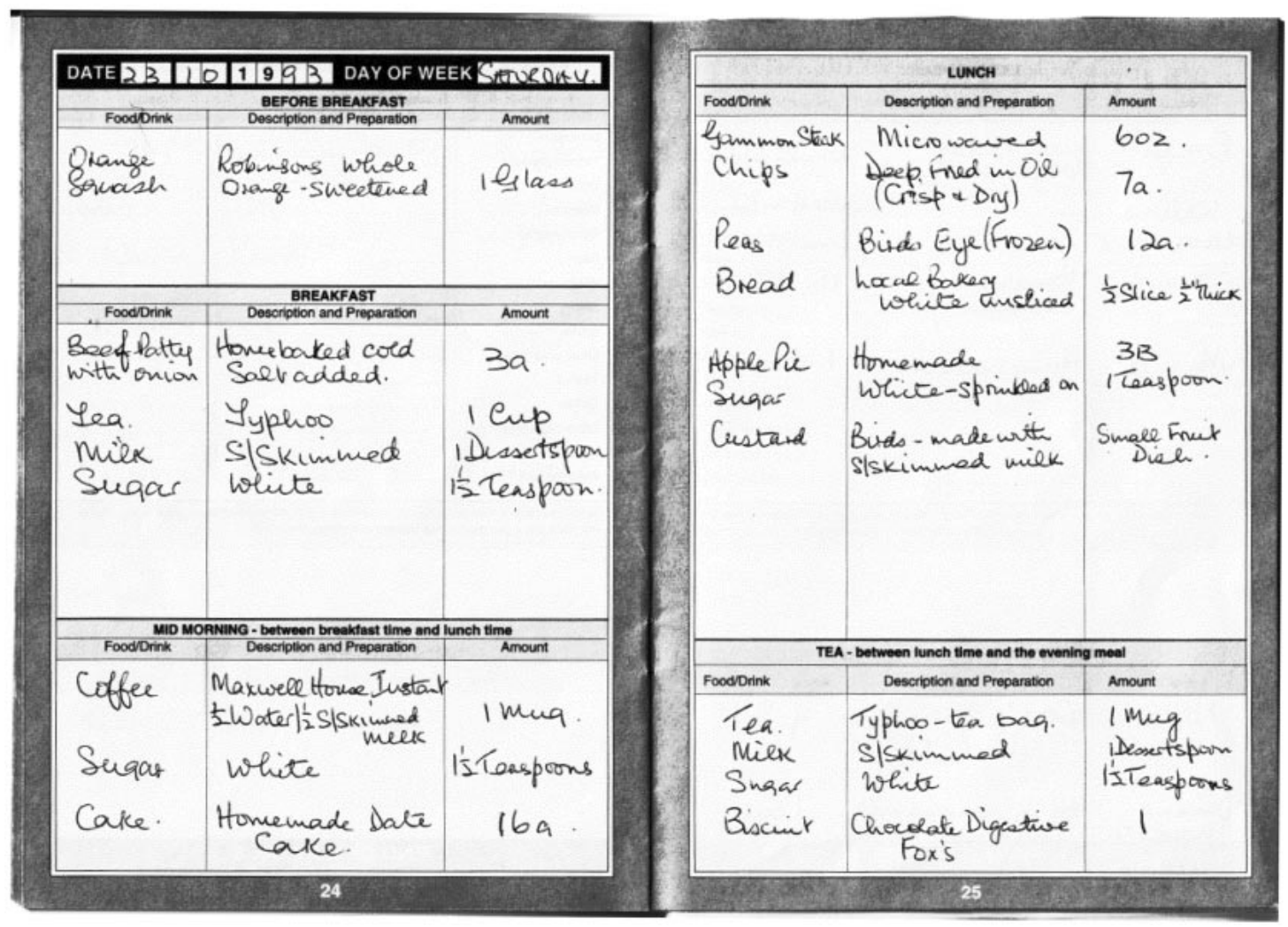

Fig. 1 Example of reported 7D food diary data

of the complete DINER system, including definitions used, is illustrated in Fig. 2 (stages A to H).

The DINER program is written in the window-based programming language TCL/TK for multi-user access on UNIX operating systems (Fig. 2, stage B). It is hoped to make the entry module more widely available for use on LINEX systems and, providing funding can be obtained, to make the complete system available to others.

As the program is used, lists of food items, portion sizes and associated information for fat type, brand name and cooking factors appear within a series of windows.

The main program was designed as a separate module from the set of menu files on which it operates. The menu files hold the food and portion lists and associated information. They are automatically generated from a set of relational databases using food group as a central link. Menu generation is version-controlled. Old menus are archived at each menu update. The database design is an important feature and individual menu combinations are generated for each food item, so that specific sets of windows appear during entry.

The individual structured DINER files (Fig. 2, stage C and Fig. 9) are combined into the DINER database (Fig. 2, stage E). The nutrient calculation program (NCP) is separate from the entry program (Fig. 2, stage F). The NCP calculates quantities from portion descriptions and then nutrients that are incorporated into the DINER database (Fig. 2, stage E). Final calculation of individual intake of foods and nutrients occurs (Fig. 2, stage G). Check programs operate on stages C, E and G (Fig. 2).

\section{Description of the software}

\section{The entry process}

A food list item, portion size and number of portions are entered for all reported foods. The entry process entails making choices from a series of windows to produce the final data window as illustrated in Figs 3-9.

An identification number (with check sum digit), the diary version and the date of record are entered for everyone. The software and menu versions, coder identification code and date of entry are automatically assigned within the data structure (Fig. 9a, columns A, B and E).

\section{Copy and paste}

A 'copy and paste' function allows items already entered to be copied to another meal slot or day, and is used extensively for beverages and other routinely consumed items.

\section{Factors affecting intake}

Food diaries are entered according to meal slot. Reasons for missing meals are documented and factors affecting 

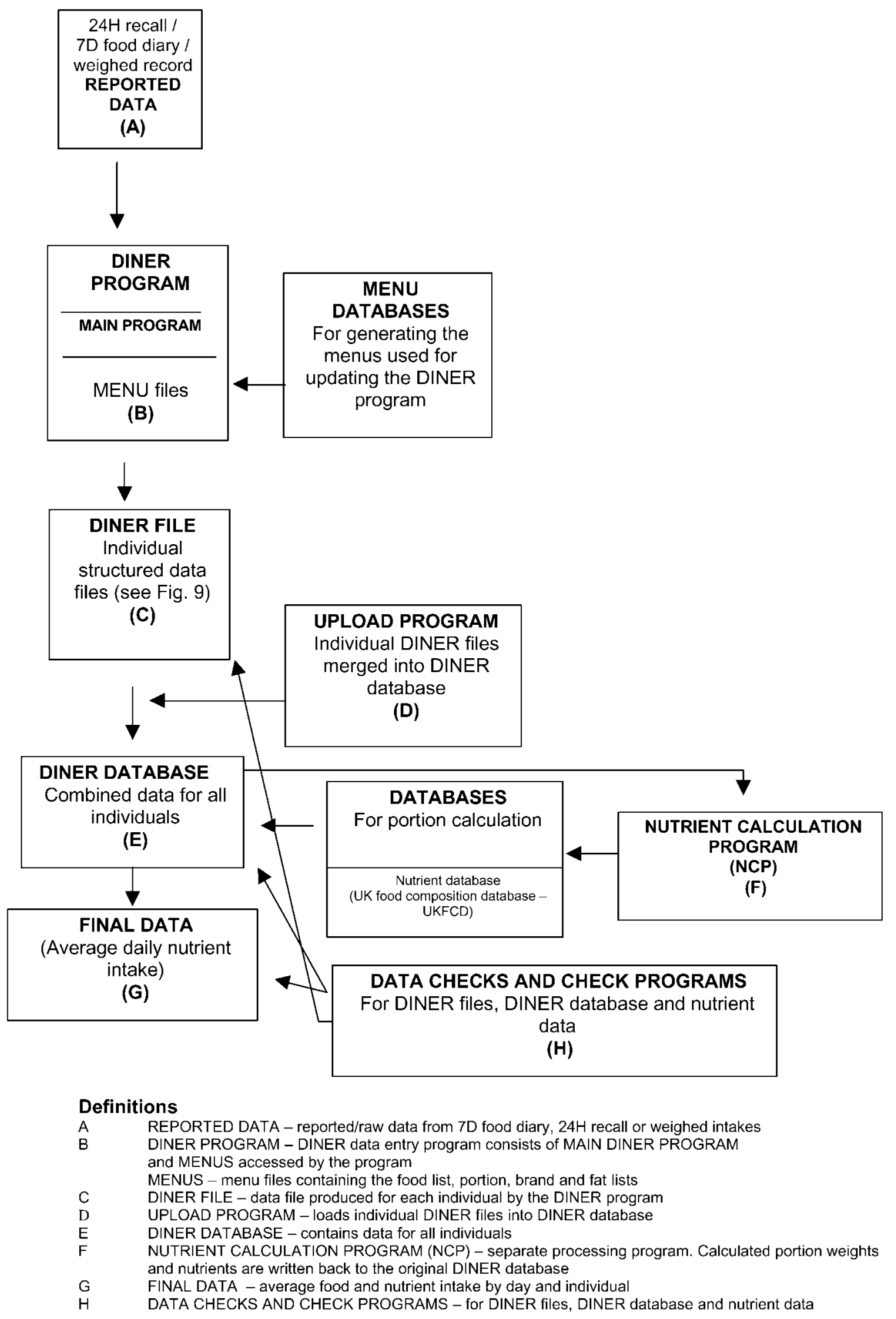

Fig. 2 Schematic representation of the DINER system with associated processes and definitions

usual intake such as illness or special diet are recorded and may help to explain differences in energy intakes (Fig. 3).

\section{Food list}

Around 150000 food items exist within the UK food supply, but only 3000 are listed within the UK food composition database (UKFCD) $)^{8-17}$. The food list had to take into account the existing requirements for the study and predictions of future hypotheses, and was constructed by review of each major food group. The level of 


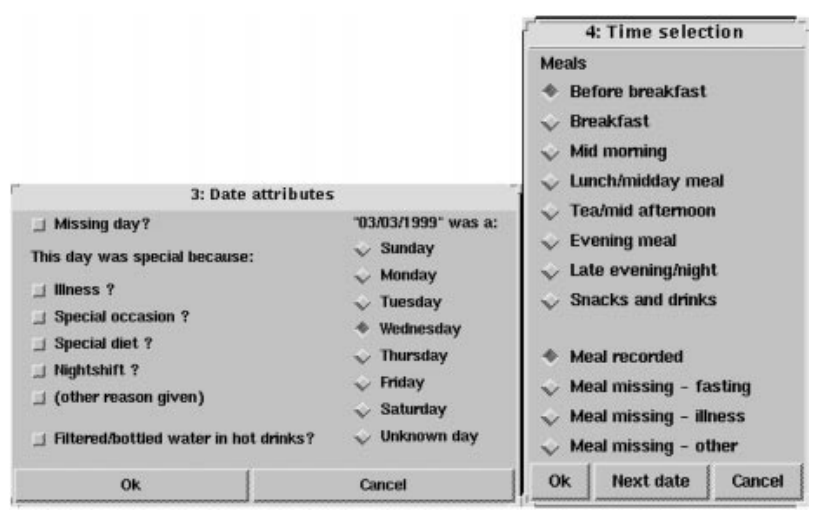

Fig. 3 Date and time selection

classification of the foods was decided in advance so that when new food items appeared they could be included in the list without change to the existing items. Home-made and commercial versions of food items were included where the composition varies systematically. The list distinguishes between foods containing phyto-nutrients such as carotenoids and phyto-oestrogens and nonnutrients such as the heterocyclic amines formed during the cooking of meat.

Items classified at different levels of specificity are included in the list to aid consistency of choice and entry at the level of reported detail (see Fig. 5). Apple pie may be entered using one of the choices illustrated in Fig. 5, but if no information on the type and proportion of pastry is given, the item apple pie n.s. (non-specific) is chosen. Items consisting of mixed single components such as salads or sandwich fillings are included in the list to avoid arbitrary estimation of quantities.

\section{Food selection}

Foods are selected by using the food-group-based set of hierarchical menus or the search string mechanism (Figs 4 and 5). The software and food name structure have been designed for rapid and efficient use of search mechanisms.

The string search utilises strings (identified as a series of letters) that may be part or the whole of a food name to select all food items containing a particular string. Searches can retrieve items from the overall level or at any point below this at group or sub-group level. Sequential nested searches can be made. The search options available are shown in Fig. 5. Combinations of two or more strings are the most rapid method of locating specific items. The search mechanism operates on all menus for portion size, fat type and brand name.

\section{Food name structure}

The food name structure was devised so that names are clear, consistent and understandable for accurate and rapid location and clear differentiation during entry. The name length of 100 characters allows adequate detail including synonyms, brand information and equivalent food types to be included. The structure consists of a main name, e.g. apple pie, followed by a first additional name and further descriptions as necessary, e.g. wholemeal and individual/one crust.

Consistency of naming ensures that the results of searches appear in meaningful order. For instance, in a search on the strings 'apple pie', versions prepared with wholemeal pastry appear at the end of the list (see Fig. 5).

\section{Food portion estimates}

Once a food item is chosen, a group-specific list of portion sizes appears (Fig. 7). A complete 'generic' list of portions is also accessible. The number of portions is entered. In Fig. 6, one portion of photo $3 \mathrm{~b}$ was chosen

For foods consumed in the cooked form but sometimes reported as the uncooked weight, such as meat and rice, an additional window appears so that the portion can be described as uncooked.

\section{Fat in food preparation}

Information on fat type, brand and description of recipes is recorded for certain foods. A menu requesting a choice of fat appears for foods in the groups that contribute to the majority of fat in the UK diet, namely fried foods,

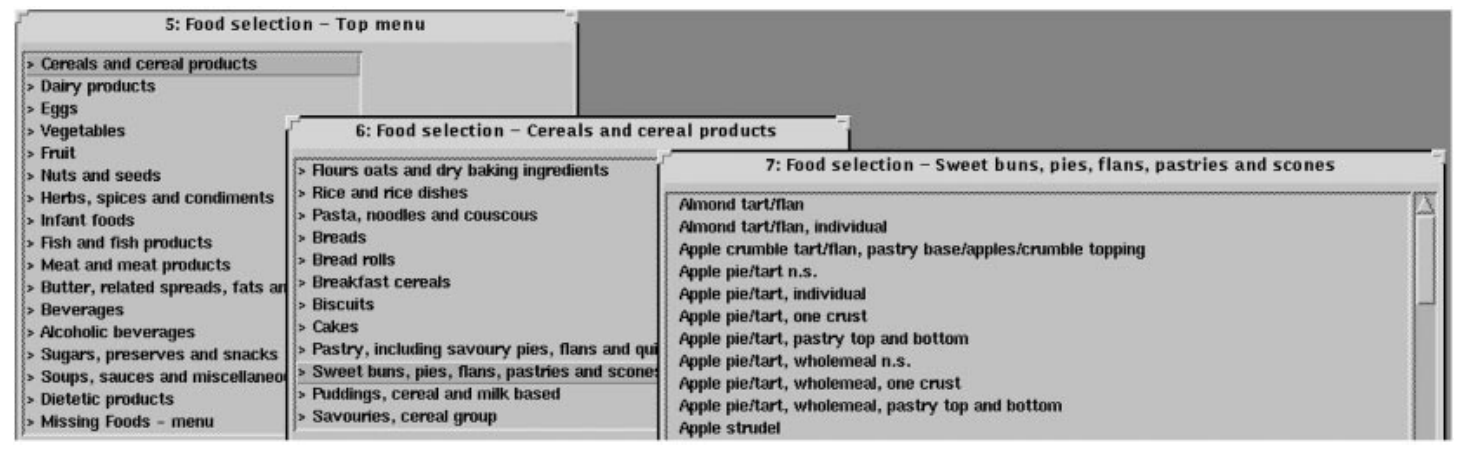

Fig. 4 Selection of apple pie via the menus 


\begin{tabular}{|c|c|c|}
\hline \multicolumn{3}{|c|}{ 6: Food selection - full search on "apple pie" } \\
\hline \multicolumn{3}{|c|}{$\begin{array}{l}\text { Apple pie/tart n.s. } \\
\text { Apple pie/tart, individual } \\
\text { Apple pie/tart, one crust } \\
\text { Apple pie/tart, pastry top and bottom } \\
\text { Apple pie/tart, wholemeal n.s. } \\
\text { Apple pie/tart, wholemeal, one crust } \\
\text { Apple pie/tart, wholemeal, pastry top and bottom }\end{array}$} \\
\hline \multicolumn{3}{|c|}{ Enter search string(s): [} \\
\hline $\begin{array}{l}\text { All strings ... } \\
\text { Case sensitive? }\end{array}$ & $\begin{array}{l}\text {... WITHOUT spaces ... } \\
\text { If Starts word? } \\
\text { Ends word? } \\
\text { if Starts whole name? }\end{array}$ & $\begin{array}{l}\ldots \text { WITH spaces } \\
\text { all strings? } \\
\text { any string? } \\
\text { match as typed! }\end{array}$ \\
\hline Ok & & Cancel \\
\hline
\end{tabular}

Fig. 5 Food selection - full search on apple pie

home-made recipes, commercial dishes, and cakes and biscuits. Up to two types of fat may be chosen (see Figs 7, 8 and $9 \mathrm{a}$, columns $\mathrm{X}$ and $\mathrm{Y}$ ). Where relevant, the brand and place of preparation, e.g. home-made or commercial (as this also affects the composition of fat), are recorded via the brand attribute. Programs will be developed to incorporate the type of fat into calculations of fatty acid consumption.

\section{Brand names}

Different brands of the same food type can contain varying levels of fat, energy or nutrient fortification but

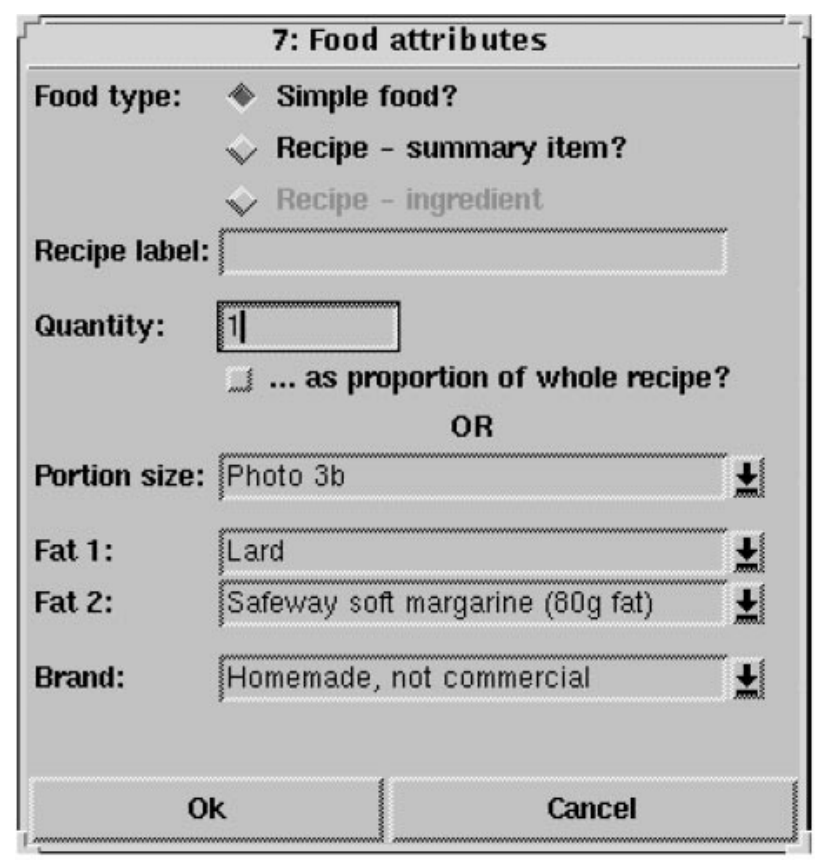

Fig. 6 An example of chosen food attributes prior to addition to the DINER file

\begin{tabular}{|c|c|c|}
\hline \multicolumn{3}{|c|}{ 8: portion selection } \\
\hline $\begin{array}{l}\text { Finger } \\
\text { g/gram } \\
\text { Individual c } \\
\text { Individual c } \\
\text { Individual c } \\
\text { Individual c } \\
\text { Individual c } \\
\text { Individual p } \\
\text { Individual p } \\
\text { Individual p } \\
\text { Individual p } \\
\text { Individual p } \\
\text { Individual t } \\
\text { Individual t } \\
\text { Individual t } \\
\text { Individual t } \\
\text { Individual t } \\
\text { ozfounce } \\
\text { Photo 3a } \\
\text { Photo } 3 \mathrm{~b} \\
\text { Photo 3c } \\
\text { Portion (for } \\
\text { Portion/sen } \\
\text { Portion/ser } \\
\text { Portion/ser }\end{array}$ & ed) & K \\
\hline $\begin{array}{l}\text { Enter searc } \\
\text { portion file } \\
\diamond \text { Specif } \\
\diamond \text { Generi }\end{array}$ & & \\
\hline OK & Cancel & \\
\hline
\end{tabular}

Fig. 7 Portion selection menu

the UKFCD contains little specific information for commercial foods. Brand is recorded for foods in the groups contributing the most to fat or energy, or where the nutrient fortification levels are variable, for future re-classification (Fig. 6).

\section{Composite or recipe foods}

Recipe foods (or composite dishes) vary in the number, type and proportion of ingredients used to prepare them. The UKFCD contains a number of the most commonly consumed dishes. This list has been revised and extended to cover the range of dishes and nutritionally important ingredients commonly consumed.

During entry all reported recipes are assigned to a 


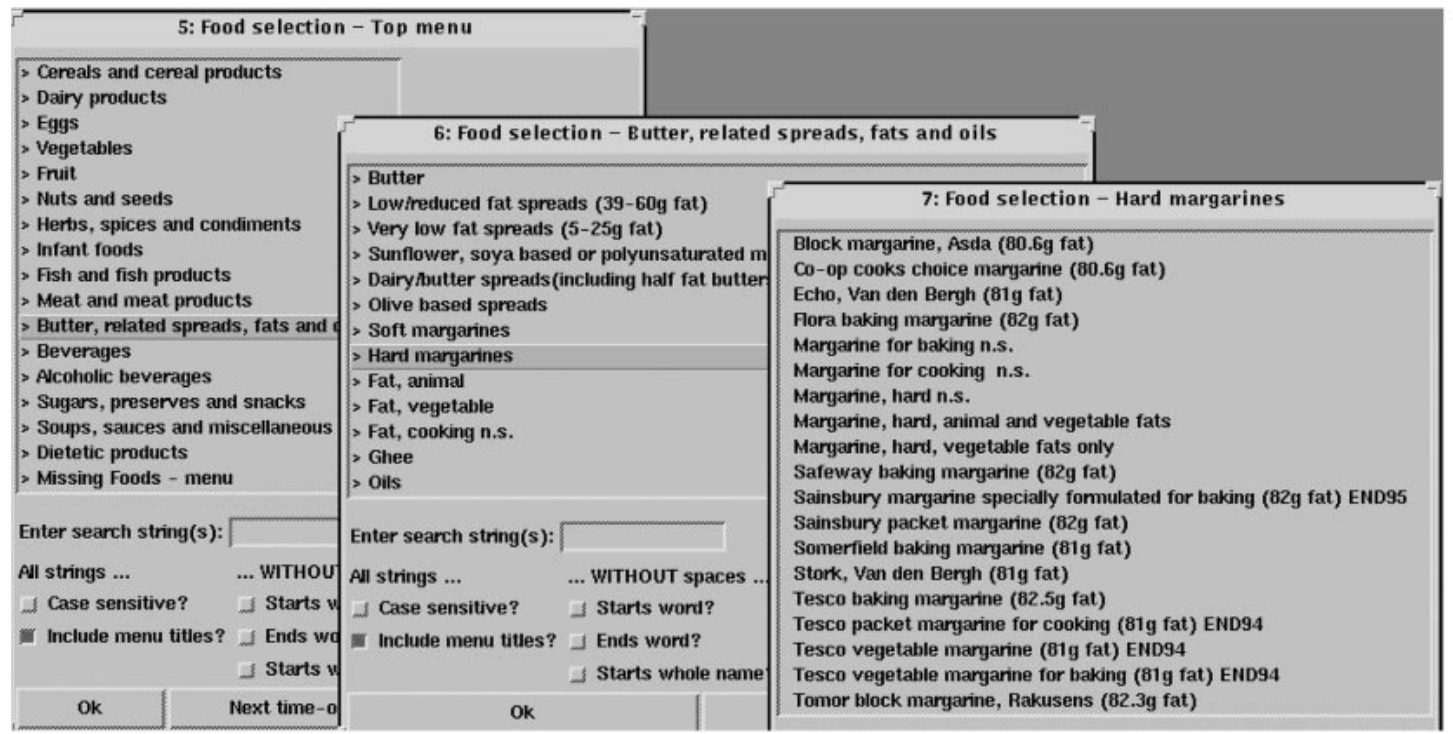

Fig. 8 Fat selection via menu

standard recipe. Where individuals have reported details of ingredients for recipes, these are summarised, in text form, in the recipe label field. This stores the information for future use and allows review of recipe items.

\section{Data structure}

Data are written to the DINER file once all decisions have been completed. Each food is represented by one line of data in the final structure, see Fig. 9. A simplified view of this data structure is used during entry.

\section{Staff training, issues of consistency and problem solving}

It is important that consistency should be achieved between coders and coding on different occasions.

Data are entered for specific sub-studies and coders are unaware of the purpose. The same coders enter data for cases and controls to ensure consistency.

New entry staff undergo a two-day training programme with a qualified nutritionist (AMcT). Intensive support, checking and feedback are given until the level of knowledge of, and familiarity with, the program and entry protocol is considered adequate.

Decisions and issues of interpretation that have been made during the development and use of DINER have been documented in a 177-page reference manual, to ensure a consistent approach to entry. This includes the priorities to be used in the decision process where the reported data are incomplete. New decisions are updated within the manual. On a day-to-day basis one person is responsible for decisions regarding entry.

Trained coders identify problems and use the reference manual for backup. Issues not readily solved are referred to a nutritionist for solution. Food items requiring further investigation are entered as the next best match on the food list and identified as difficult to categorise (DCI) for later checking. On average a nutritionist deals with two queries per diary, one of which can usually be resolved immediately.

A reference bank of information for commercial food products was instigated at the start of the study and is kept up-to-date for consultation.

\section{New foods}

After completion of a batch of diaries, the DCI are identified and the nutritional composition evaluated. This requires review of the available information and possible contact with food manufacturers and retailers. Where possible, DCI are entered using existing food list items. Items that are sufficiently different are added to the food list as new foods. The existing DINER data are then edited.

Foods considered to be new fit one or more of the following criteria: a new food type; nutritionally different from other items within a food group; or a mixed dish prepared by an established cooking method. This same approach is used to evaluate food portion descriptions, fat types and brand names.

\section{Conversion into mutrients and food groups}

Individually created files are amalgamated into the DINER database (stages D and E in Fig. 2). A separate nutrient calculation program (NCP) calculates firstly portion sizes and secondly nutrients. These values and a number of intermediate calculations and factors are read back into the DINER database (columns AJ-AS and BB-BD, Fig. 9b).

The nutrient database used for calculations is based on 2200 foods in the UKFCD ${ }^{8-17}$; mainly the supplements to McCance and Widdowson's The Composition of Foods. 

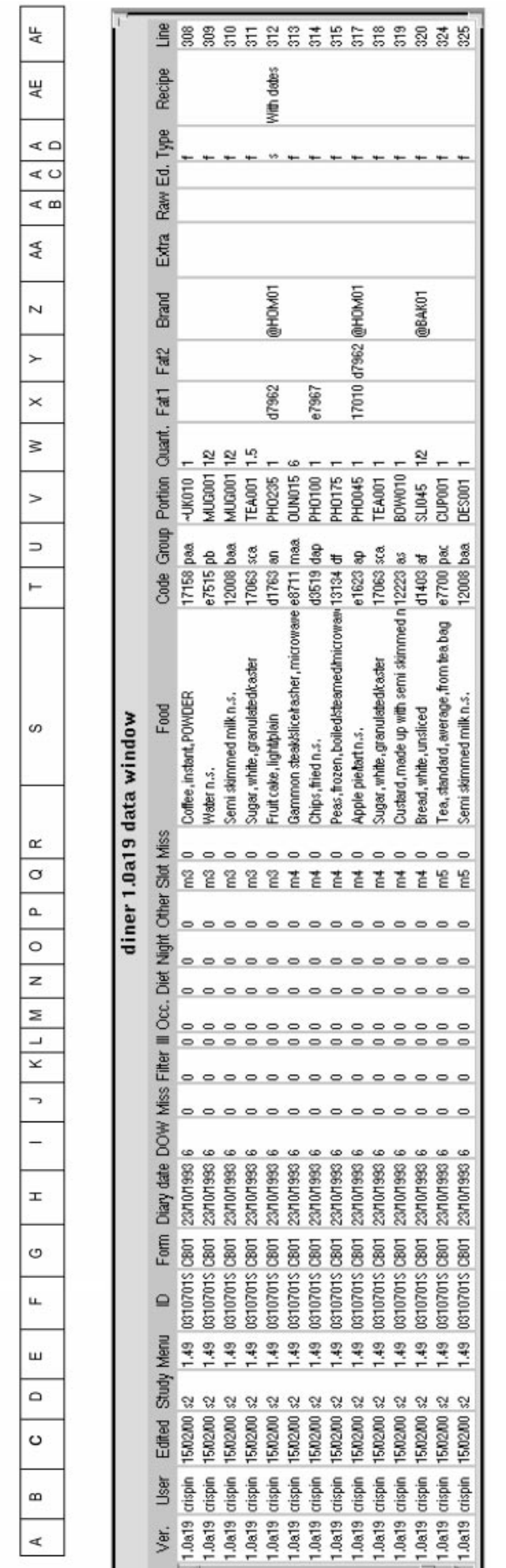

के

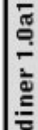

密

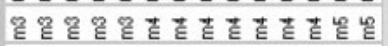

00000000000000

言

00000000000000

O.00000000000000

要 00000000000000

$\frac{1}{2} 00000000000000$

00000000000000
00000000000000

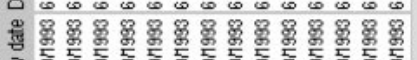

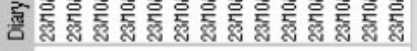

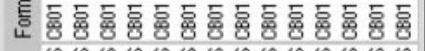

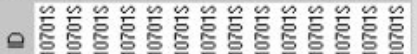

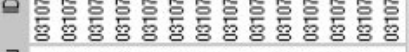

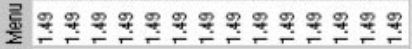

营

总

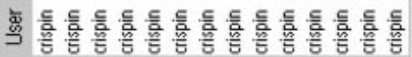

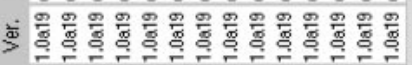

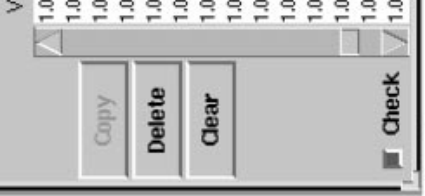
要

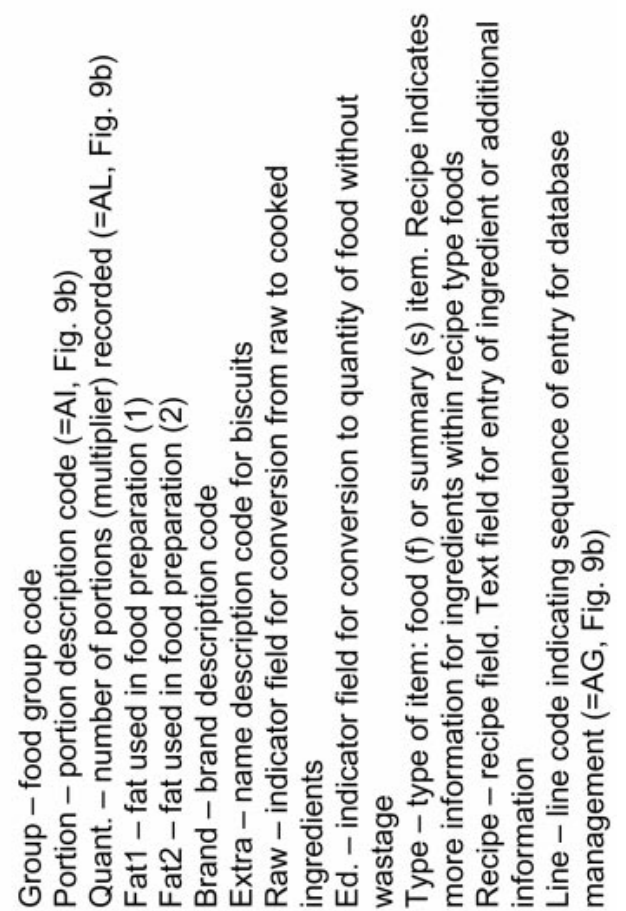

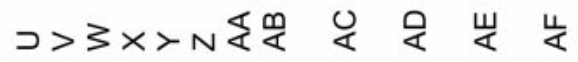

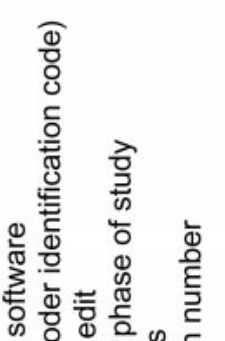

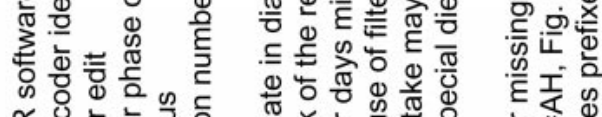

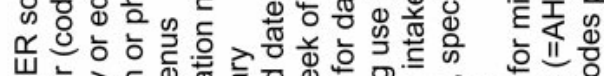

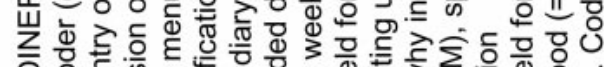

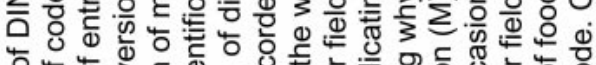

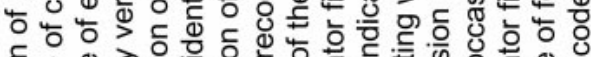

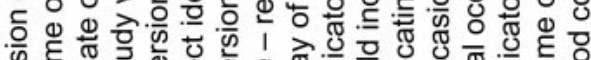

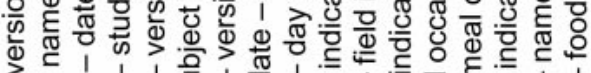
$>111110$

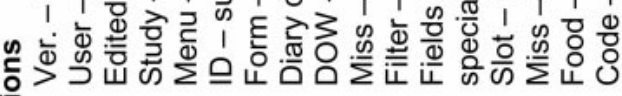

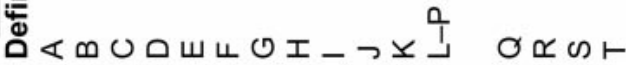




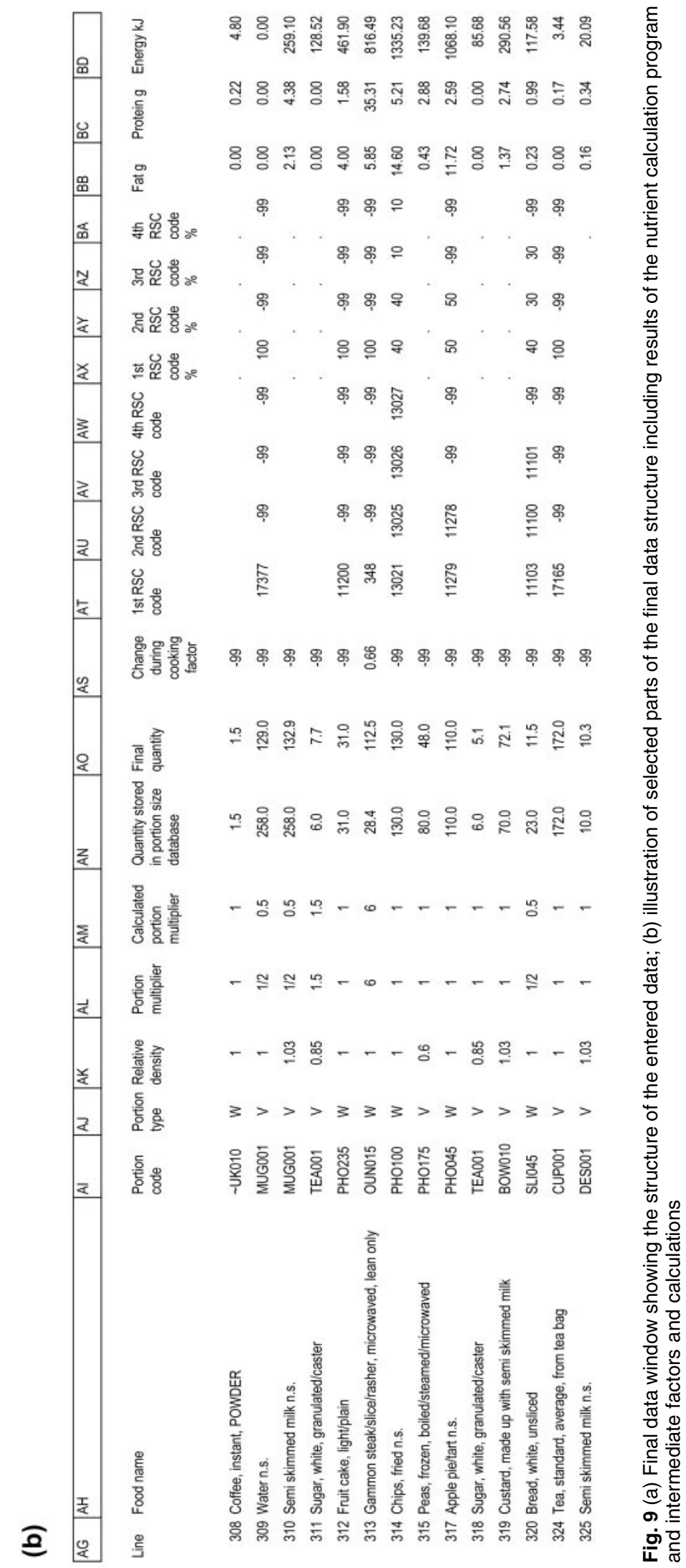


For new foods, nutrient data are derived by matching the known composition of the new food to proportions of up to four food items in the UKFCD, to create the best possible nutritional equivalent. A set of criteria has been developed to guide the decisions of the mapping process and take into account the important nutrients for each group. This system accounts for the different composition of new foods, ensures consistent treatment and utilises validated official data. The NCP applies a proportion of each UKFCD code (Fig. 9, columns AT-BA). Apple pie n.s. (code e1623) is represented by 50\% each of UKFCD codes 11279 and 11278 (apple pie with one crust and apple pie with pastry top and bottom).

As the UKFCD is considerably incomplete for some nutrients, a series of in-filling programs is ongoing. The design of DINER will allow the range of nutrients available to be extended.

\section{Portions}

Portions described as either weight or volume are converted via a number of databases to gram weight values. The main portion database has been structured for efficiency so that portion size values are applied at different levels to all foods, particular sub-groups or specific foods.

Conversion factors for relative density or to account for wastage during food preparation are applied to specific foods to reach the final gram weight (columns AK-AO, Fig. 9b). For example, the value for peas is derived by converting the representative volume for photo $12 \mathrm{a}$ $(80 \mathrm{ml})$ by the relative density of 0.6 and multiplied by 1 to give the final quantity of $48 \mathrm{~g}$.

Certain foods estimated by weight of the uncooked form are converted to the cooked weight equivalent. An example is gammon steak, shown in Fig. 9b, converted using the factor in column AS to reach the final weight $(6 \times 28.4 \times 0.66=112.5 \mathrm{~g})$.

As new foods (see below) are added to the food list, new portions are identified. Default values are applied in the first instance where portion sizes are missing. Inappropriate portion choices are systematically converted to more correct choices.

Consumption of foods by group is computed separately.

\section{Data checks}

A series of post-entry checks ensures correct data are available for analysis. The data structure has allowed the development of automated checks to replace some manual checks and reduce data handling (Fig. 2, stage H).

The design of DINER limits the capacity to enter incorrect data. Data are chosen from fixed lists and written to file only after choices are complete. Errors that do occur may be due to incorrect choice or combination of foods or portion sizes, or to incorrect numbers of portions. Foods may also be missed, duplicated or subject to misinterpretation errors.

For new entry staff, preliminary checks are made routinely by a more experienced coder by comparing the original record with a printout of the entered data. A further program is run on each diary to check for inappropriate food and portion combinations, discrepant dates and completion of all meal slots. Meals that are smaller than expected, i.e. containing fewer than two items, are identified, as are duplicates of main meal type food items, e.g. meat dishes. The data are screened to ensure the number of portions is correctly configured and unexpectedly high numbers of portions are checked. Incorrect values are edited.

An automated check on nutrient output identifies maximum expected values for individual foods and minimum and maximum values for expected nutrient intake per day. For the volume of data generated by EPIC, this process would previously have required visual inspection by several nutritionists.

\section{Test for consistency of data entry}

The impact of the attempt to achieve consistency of entry has been evaluated using energy intake. The hypothesis tested was that age, sex, height, weight and batch of entry (dataset) would be the major determinants of energy intake and the inclusion of a variable to define coder would not have a significant impact in a multivariate regression model.

Data used for the analysis included those diaries entered for descriptive population studies, cases of nonfatal disease and controls. The 25 coders who had entered 15 or more diaries were included and, after exclusion for missing data, this resulted in 3525 individuals available for analysis. Dataset was added to the model to account for entry of data in different batches. Three multivariate regression models were tested on the dependent variable energy intake $(\mathrm{kJ})$. The first included sex, age, height and weight. The second also included dataset. The final model included coder in addition to the other covariates. Tests for maximum likelihood were performed between each model.

Mean energy intake was also calculated for each entry person and adjusted for sex, age, weight, height and dataset. Analyses were performed using STATA V6.0.

\section{Results}

\section{Data collected}

Ninety-two per cent of participants completed and returned their first 7D diary. A total of $504657 \mathrm{D}$ diaries (23 655 first, 15666 second and 11144 third diaries) and 52363 self-reported 24H were collected between 1993 and 2000. This provides 21 days of reported intake for 
$47 \%$ of the population who completed three diaries for later analysis of repeatability and seasonal variation.

Five thousand nine hundred and seventy-nine diaries and $82424 \mathrm{H}$ recalls have been entered using DINER since 1995, a total of 1251000 lines of data. Thirty-four staff have been trained. Each diary averages 210 lines of data. Mean entry time was 75 min per diary (range $0.75-3.5 \mathrm{~h}$ ). After the initial training stages, support of entry was around 5 min per diary. Further processing, checking and data management took around $1 \mathrm{~h}$ per diary.

\section{Food list}

The compiled food list presently consists of 9000 items, $30 \%$ of which are directly equivalent to the UKFCD. The remainder are new food items that have been included to guide entry, provide specific classifications for data analysis and to account for the composition of foods not available within the UKFCD. The food list includes more specific detail for foods of particular nutritional importance in the UK such as breads, breakfast cereals and fat spreads. So far, 337 specific types and brands of breakfast cereals have been identified and mapped to the 32 items within the UKFCD.

\section{Food portions}

The database contains 24000 values compiled from official publications $(26 \%)^{18,19}$, obtained from food manufacturers or retailers (35\%), or imputed from established sources. There are 3200 density, edible part and cooking loss factors stored in the databases used by the NCP. Values for these factors were derived from inhouse measurements or published sources ${ }^{8-14,16,17}$.

\section{Brand names}

The brand name database contains 900 brands and brand types.

\section{Food groups}

Two hundred and forty-two food groups have been developed for classification of the food list, menu generation program and data entry.

\section{Nutrient and food intake}

Mean intake of nutrients and food groups produced by the DINER system have been presented elsewhere ${ }^{20}$.

\section{Consistency of entry}

For the analysis of consistency of entry, the number of diaries entered by each coder is shown in Table 1. For model 1 all of the covariates were significantly related to energy intake: sex, $\beta=1478(P<0.001)$; weight, $\beta=$ $-15 / \mathrm{kg}(P<0.001)$; height, $\beta=61 / \mathrm{cm}(P<0.001)$; and age, $\beta=-30 /$ year $(P<0.001), r^{2}=0.308$. The addition of dataset to model 2 increased $r^{2}$ to 0.317 . The addition of coder to model 3 increased $r^{2}$ to 0.325 . A maximum likelihood ratio test confirmed that the effect of adding coder to the model was significant $(P<0.013)$. Three
Table 1 Number of diaries entered by individual coders

\begin{tabular}{lrc}
\hline Coder & Number & Percentage \\
\hline 1 & 88 & 2.5 \\
2 & 81 & 2.3 \\
3 & 39 & 1.1 \\
4 & 37 & 1.1 \\
5 & 118 & 3.4 \\
6 & 22 & 0.6 \\
7 & 249 & 7.1 \\
8 & 528 & 15.0 \\
9 & 32 & 0.9 \\
10 & 145 & 4.1 \\
11 & 39 & 1.1 \\
12 & 307 & 8.7 \\
13 & 499 & 14.2 \\
14 & 205 & 5.8 \\
15 & 164 & 4.7 \\
16 & 84 & 2.4 \\
17 & 36 & 1.0 \\
18 & 79 & 2.2 \\
19 & 112 & 3.2 \\
20 & 164 & 4.7 \\
21 & 124 & 3.5 \\
22 & 114 & 3.0 \\
23 & 84 & 2.4 \\
24 & 97 & 2.8 \\
25 & 78 & 2.2 \\
& 3525 & 100 \\
\hline & &
\end{tabular}

coders showed the greatest impact on entry: numbers 14, $\beta=-1293(P<0.015) ; 19, \beta=-1253(P<0.026)$; and 5, $\beta=-1223(P<0.015)$. When these were dropped from the analysis and the model re-run and tested for maximum likelihood, the effect of coder was no longer significant $(P<0.122)$, indicating that consistency had been achieved for the remaining 22 coders.

Means of energy intake were adjusted for age, height and dataset for each coder. The deviation from the group mean is illustrated in Fig. 10 for women. The $X$-axis indicates the number of diaries entered by each coder. Deviation from the mean is generally lower in those entering more diaries. It is greatest in those entering data at the start of the study.

\section{Discussion}

Around 150000 food items exist within the UK food supply and individuals may report and describe any of them with varying levels of adequacy and precision. Furthermore, the vast changes that have affected food supply in recent years are set to continue. Around 10000 foods are modified, appear or are discontinued each year. Ranges of pre-prepared meals have increased and new and functional foods have appeared in the market place. Ordinary foods have been fortified with additional nutrients and existing foods reduced in macronutrient density; mainly sugar and fat. Such diversity and change represents an almost overwhelming problem in nutritional epidemiology. However, DINER is capable of tracking changes in the food supply by accommodating 


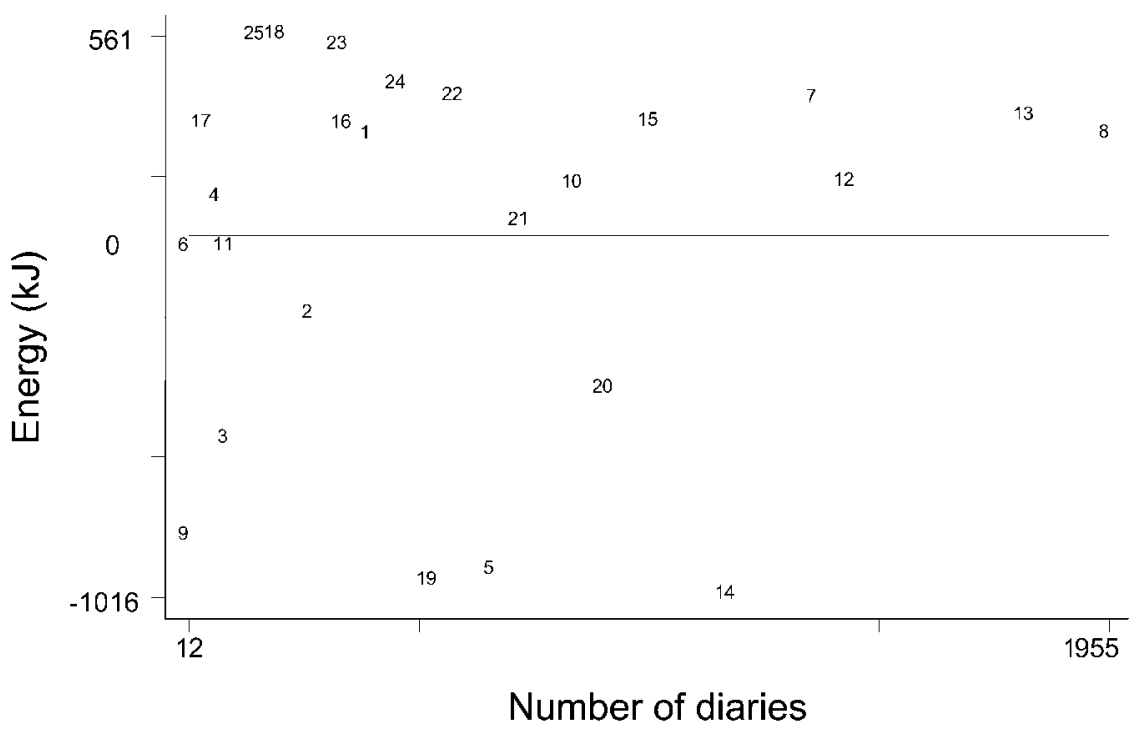

Fig. 10 Plot of difference from mean energy $(\mathrm{kJ})$ in women (adjusted for age, height, weight, sex and dataset) by individual coders. Ordered by number of diaries entered ( $X$-axis)

those that affect nutrient composition. The detailed classification structure for food items allows new foods to be incorporated without the need to change existing items. New portions, fat types and brand names can also be added. The information garnered from monitoring change to the food supply over six years has been incorporated.

Some nutritional knowledge to distinguish the relevant characteristics when interpreting and matching reported foods with those in DINER is needed. The features of a detailed, systematic food classification and name structure reduce reliance on pre-existing nutritional knowledge. The detailed food and portion lists speed up and increase the accuracy of entry and the ability to locate items, and reduce ambiguity and the onus for decision making by individual coders. The food list acts as the interface to avoid misclassification. This is particularly important in groups such as breakfast cereals, in which non-starch polysaccharides, fat, minerals, vitamins and sugar vary widely. The use of consistent defaults for inadequately reported foods, portions, mixed component foods and new food items also reduces the burden of interpretation and potential for bias between coders.

The detailed, extended food names reduce the requirement for external documentation and look-up lists for equivalent foods. In contrast, traditional entry systems require coders to memorise equivalent items with the associated potential for incorrect choice and bias.

The level of detail incorporated in DINER eliminates much of the possible bias between coders, and the results of the analysis for consistency indicate that this has been achieved for the majority (Fig. 10). The least consistent coders were involved early in the use of the program, prior to the development of the extensive entry guidelines.
It is possible that we have not completely accounted for all of the factors that influence consistency of entry in the analytical model.

The development of automated checks using the data structure allows more checks than other systems. However, this advantage means an almost infinite amount of time can be spent on data correction. Hence, the relative impact of proposed modifications or corrections is evaluated.

Familiarity with the software soon leads to knowledge of the most useful combination of menu level and search string for rapid selection of food items. Entry and use of the extensive check programs takes $2 \mathrm{~h}$ per diary and results in clean data ready to use for analysis without further handling. The introduction of immediate post-entry checks should reduce this time further.

It was anticipated that nutritional assessment would be more accurate if reported recipes were calculated from individual ingredients rather than using the existing standards and the original DINER specification included a method for this. However, a review of entered diaries revealed that only $4 \%$ of items were individual recipes and many were incomplete. Hence a recipe module was not included. Further development will involve partitioning the contribution of individual foods, such as onions or tomatoes, to estimate foods found within recipes.

Separation of the main program from menu generation means that change can be implemented without the high cost of developing new entry programs. Entry of data as close to the original description, non-hard coding of actual values for foods and portion sizes, and separation of the NCP, ensure flexibility and potential for updating. The NCP acts on modifiable databases, making it possible to change any database value and re-run the program 
without recourse to editing the DINER files. New versions of the UKFCD will be incorporated.

Measures have been taken to improve the accuracy of the data collection of the 7D diary by ensuring it was as complete as possible and not subject to interviewer bias. The method of matching the composition of new foods to UKFCD items addresses the shortfall in information in the food composition tables. The rationale for the development of DINER has balanced the known limitations of food record methods, e.g. the limited accuracy of portion estimates $^{21}$, and the requirements of flexibility and accuracy of the final data. The need to satisfy the multiple hypotheses generated by a multiple-disease cohort study was anticipated and the detailed food classification structure allows specific variables to be created for analysis. For instance, the intake of brassica vegetables acts as a surrogate for glucosinolate consumption.

In conclusion, accurate methods for measuring dietary intake are needed in large cohort studies to minimise random and systematic measurement errors that may reduce power or bias results. Data-entry methods that can cope with the rapidly changing food supply and extensive variety of foods consumed are required. The development of DINER has addressed a number of the problematic issues that can impede the use of $7 \mathrm{D}$ diaries for large prospective cohort studies and has made the use of such data feasible within EPIC-Norfolk. DINER is a flexible system, capable of being updated and of accommodating change for use in prospective dietary surveys and, to our knowledge, is the first in the UK to adopt this type of approach.

\section{Acknowledgements}

We thank the participants and general practitioners who took part in the study and the staff of EPIC-Norfolk. EPIC-Norfolk is supported by research programme grant funding from the Cancer Research Campaign and the Medical Research Council, with additional support from Stroke Association, British Heart Foundation, Department of Health, Europe Against Cancer Programme Commission of the European Union, the Food Standards Agency and the Wellcome Trust.

\section{References}

1 Bingham SA, Gill C, Welch A, et al. Validation of dietary assessment methods in the UK arm of EPIC using weighed records, and 24-hour urinary nitrogen and potassium and serum vitamin $\mathrm{C}$ and carotenoids as biomarkers. Int. J. Epidemiol. 1997; 26(Suppl. 1): 137-51.

2 Day NE, McKeown N, Wong MY, Welch AA, Bingham SAB. Epidemiological assessment of diet: a comparison of a $7-$ day diary with a food frequency questionnaire using urinary biomarkers of nitrogen, potassium and sodium. Int. J. Epidemiol. 2001; 30: 309-17.
3 McKeown NM, Welch AA, Runswick SA, et al. The use of biological markers to validate self-reported dietary intake in a random sample of the European Prospective Investigation into Cancer (EPIC) UK, Norfolk cohort. Am. J. Clin. Nutr. 2001 [in press].

4 Kipnis V, Midthune D, Freedman LS, Bingham SA, Schatzkin A, Subar A, Carroll RJ. Empirical evidence of correlated biases in dietary assessment instruments and its implications. Am. J. Epidemiol. 2001; 153(4): 394-403.

5 Riboli E. Nutrition and cancer: background and rationale of the European Prospective Investigation into Cancer and Nutrition (EPIC). Ann. Oncol. 1992; 3(10): 783-91.

6 Day NE, Oakes S, Luben R, et al. EPIC-Norfolk: study design and characteristics of the cohort. Br. J. Cancer 1999; 80(Suppl. 1): 95-103.

7 Braddon FE, Wadsworth ME, Davies JM, Cripps HA. Social and regional differences in food and alcohol consumption and their measurement in a national birth cohort. $J$. Epidemiol. Community Health 1988; 42(4): 341-9.

8 Holland B, Welch AA, Unwin ID, Buss DH, Paul AA, Southgate DAT. McCance and Widdowson's The Composition of Foods, 5th ed. Cambridge: Royal Society of Chemistry, 1991.

9 Holland B, Unwin ID, Buss DH. Cereals and Cereal Products. Third Supplement to McCance and Widdowson's The Composition of Foods, 4th ed. Cambridge: Royal Society of Chemistry, 1988.

10 Holland B, Unwin ID, Buss DH. Milk Products and Eggs. Fourth Supplement to McCance and Widdowson's The Composition of Foods, 4th ed. Cambridge: Royal Society of Chemistry, 1989.

11 Holland B, Unwin ID, Buss DH. Vegetables, Herbs and Spices. Fifth Supplement to McCance and Widdowson's The Composition of Foods, 4th ed. Cambridge: Royal Society of Chemistry, 1992.

12 Holland B, Unwin ID, Buss DH. Fruit and Nuts. First Supplement to McCance and Widdowson's The Composition of Foods, 5th ed. Cambridge: Royal Society of Chemistry, 1992.

13 Holland B, Welch AA, Buss DH. Vegetable Dishes. Second Supplement to McCance and Widdowson's The Composition of Foods, 5th ed. Cambridge: Royal Society of Chemistry, 1992.

14 Holland B, Brown JB, Buss DH. Fish and Fish Products. Third Supplement to McCance and Widdowson's The Composition of Foods, 5th ed. Cambridge: Royal Society of Chemistry, 1993.

15 Chan W, Brown JB, Buss DH. Miscellaneous Foods. Fourth Supplement to McCance and Widdowson's The Composition of Foods, 5th ed. Cambridge: Royal Society of Chemistry, 1994.

16 Chan W, Brown J, Lee SM, Buss DH. Meat, Poultry and Game. Fifth Supplement to McCance and Widdowson's The Composition of Foods, 5th ed. Cambridge: Royal Society of Chemistry, 1995.

17 Chan W, Brown J, Church SM, Buss DH. Meat Products and Dishes. Sixth Supplement to McCance and Widdowson's The Composition of Foods, 5th ed. Cambridge: Royal Society of Chemistry, 1996.

18 Ministry of Agriculture, Fisheries and Food (MAFF). Food Portion Sizes, 2nd ed. London: HMSO, 1988.

19 Nelson M, Atkinson M, Meyer J. A Photographic Atlas of Food Portion Sizes. London: HMSO, 1998.

20 Bingham SA, Welch AA, McTaggart A, et al. Nutritional methods in the European Prospective Investigation of Cancer in Norfolk. Public Health Nutr. 2001; 4(3): 847-58.

21 Nelson M, Atkinson M, Darbyshire S. Food photography. I: The perception of food portion size from photographs. $\mathrm{Br}$. J. Nutr. 1994; 72(5): 649-63. 